this process and may ultimately facilitate the prevention and treatment of kidney disease.

Address correspondence to: Rexford S. Ahima, University of Pennsylvania School of Medicine, Division of Endocrinology, Diabetes, and Metabolism, 712A Clinical Research Building, 415 Curie Boulevard, Philadelphia, Pennsylvania 19104, USA. Phone: (215) 573-1872; Fax: (215) 573-5809; E-mail: ahima@mail.med.upenn.edu.

1. Ogden, C.L., Yanovski, S.Z., Carroll, M.D., and Flegal, K.M. 2007. The epidemiology of obesity. Gastroenterology. 132:2087-2102.

2. Coresh, J., et al. 2007. Prevalence of chronic kidney disease in the United States. JAMA. 298:2038-2047.

3. Kramer, H., et al. 2005. Obesity and prevalent and incident CKD: the Hypertension Detection and Follow-Up Program. Am. J. Kidney Dis. 46:587-594.

4. Locatelli, F., Pozzoni, P., and Del Vecchio, L. 2006 Renal manifestations in the metabolic syndrome. J. Am. Soc. Nephrol. 17(4 Suppl. 2):S81-S85.

5. de Zeeuw, D., Parving, H.H., and Henning, R.H. 2006 Microalbuminuria as an early marker for cardiovas- cular disease. J. Am. Soc. Nephrol. 17:2100-2105.

6. Wachtell, K., et al. 2003. Albuminuria and cardiovascular risk in hypertensive patients with left ventricular hypertrophy: the LIFE study. Ann. Intern. Med. 139:901-906.

7. Sharma, K., et al. 2008. Adiponectin regulates albuminuria and podocyte function in mice. J. Clin. Invest. 118:1645-1656.

8. Kadowaki, T. 2006. Adiponectin and adiponectin receptors in insulin resistance, diabetes, and the metabolic syndrome. J. Clin. Invest. 116:1784-1792.

9. Pajvani, U.B., et al. 2004. Complex distribution, not absolute amount of adiponectin, correlates with thiazolidinedione-mediated improvement in insulin sensitivity. J. Biol. Chem. 279:12152-12162.

10. Nawrocki, A.R., et al. 2006. Mice lacking adiponectin show decreased hepatic insulin sensitivity and reduced responsiveness to peroxisome proliferatoractivated receptor gamma agonists. J. Biol. Chem. 281:2654-2660.

11. Yamauchi, T., et al. 2007. Targeted disruption of AdipoR1 and AdipoR2 causes abrogation of adiponectin binding and metabolic actions. Nat. Med. 13:332-339.

12. Ohashi, K., et al. 2007. Exacerbation of albuminuria and renal fibrosis in subtotal renal ablation mode of adiponectin-knockout mice. Arterioscler. Thromb. Vasc. Biol. 27:1910-1917.

13. Ouedraogo, R., et al. 2007. Adiponectin deficiency increases leukocyte-endothelium interactions via upregulation of endothelial cell adhesion molecules in vivo. J. Clin. Invest. 117:1718-1726.

14. Xu, A., et al. 2003. The fat-derived hormone adiponectin alleviates alcoholic and nonalcoholic fatty liver diseases in mice. J. Clin. Invest. 112:91-100.

15. Nishihara, T., et al. 2006. Effect of adiponectin on murine colitis induced by dextran sulfate sodium. Gastroenterology. 131:853-861.

16. Yano, Y., et al. 2007. Differential impacts of adiponectin on low-grade albuminuria between obese and nonobese persons without diabetes. J. Clin. Hypertens. (Greenwich) 9:775-782.

17. Looker, H.C., et al. 2004. Adiponectin concentrations are influenced by renal function and diabetes duration in Pima Indians with type 2 diabetes. J. Clin. Endocrinol. Metab. 89:4010-4017.

18. Pistrosch, F., et al. 2005. Rosiglitazone improves glomerular hyperfiltration, renal endothelial dysfunction, and microalbuminuria of incipient diabetic nephropathy in patients. Diabetes. 54:2206-2211.

19. Miyazaki, Y., Cersosimo, E., Triplitt, C., and DeFronzo, R.A. 2007. Rosiglitazone decreases albuminuria in type 2 diabetic patients. Kidney Int. 72:1367-1373.

20. Lee, M.J., et al. 2007. A role for AMP-activated protein kinase in diabetes-induced renal hypertrophy. Am. J. Physiol. Renal Physiol. 292:F617-F627.

\title{
Is Nef the elusive cause of HIV-associated hematopoietic dysfunction?
}

\author{
Frank Kirchhoff' ${ }^{1}$ and Guido Silvestri² \\ ${ }^{1}$ Institute of Virology, University of UIm, UIm, Germany. \\ ${ }^{2}$ Department of Pathology and Laboratory Medicine, University of Pennsylvania, Philadelphia, Pennsylvania, USA.
}

\begin{abstract}
HIV-associated hematological abnormalities involve all lineages of blood cells, thus implying that the virus impairs the function of early HSCs. However, the underlying mechanisms of this defect are unknown, particularly since HSCs are largely resistant to HIV-1 infection. In this issue of the JCI, Prost and colleagues show that the viral accessory protein Negative factor (Nef) plays a potentially critical role in the pathogenesis of HIV/SIV-associated hematopoietic dysfunction by affecting the clonogenic potential of HSCs (see the related article beginning on page 1765). Soluble Nef induces PPAR $\gamma$ in uninfected HSCs, thereby suppressing the expression of STAT5A and STAT5B, two factors necessary for proper HSC function. The identification of this novel activity of extracellular Nef defines a new mechanism of HIV/SIV pathogenesis and suggests that approaches aimed at increasing STAT5A and STAT5B expression may be considered in HIV-infected individuals with prominent hematological abnormalities. The results also raise the question of whether dysregulation of hematopoiesis by extracellular Nef plays a role in the development of $T$ cell immunodeficiency and the high levels of chronic immune activation associated with AIDS.
\end{abstract}

Nonstandard abbreviations used: Nef, Negative factor.

Conflict of interest: The authors have declared that no conflict of interest exists.

Citation for this article: J. Clin. Invest. 118:1622-1625 (2008). doi:10.1172/JCI35487.
HIV-1 infection causes immunodeficiency by inducing a progressive decline in number of $\mathrm{CD}^{+}$helper T cells. However, the mechanisms of HIV/AIDS pathogenesis are complex, as it is still unclear to what extent this progressive $\mathrm{CD}^{+} \mathrm{T}$ cell depletion is caused by direct killing of infected cells or rather by the indirect effects of chronic, generalized immune activation (1). Importantly, HIV infection is also associated with hematopoietic abnormalities characterized by impaired generation and function of cells of all blood lineages (i.e., red blood cells, platelets, and white blood cells). These hematopoietic dysfunctions may cause various levels of cytopenias and also contribute to the development of T cell immunodeficiency (Figure 1) (2). Since all blood cell lineages may be affected, HIV-1 infection apparently deregulates the function of early hematopoietic progenitor cells. This assumption was confirmed by studies showing that $\mathrm{CD} 34^{+}$progenitor cells from $\mathrm{HIV}$-infected patients show reduced growth and differentiation in vitro $(3,4)$.

HIV-associated hematological abnormalities seem to be dependent on the level of virus replication, as these abnormalities are severe in late-stage AIDS patients with high viremia but can be corrected by highly active antiretroviral therapy $(\operatorname{HAART})(5,6)$. The mechanisms underlying these hematological abnormalities are still obscure. While HSCs express low levels of CD4 and CCR5, 


\section{A Normal hemolymphopoiesis}

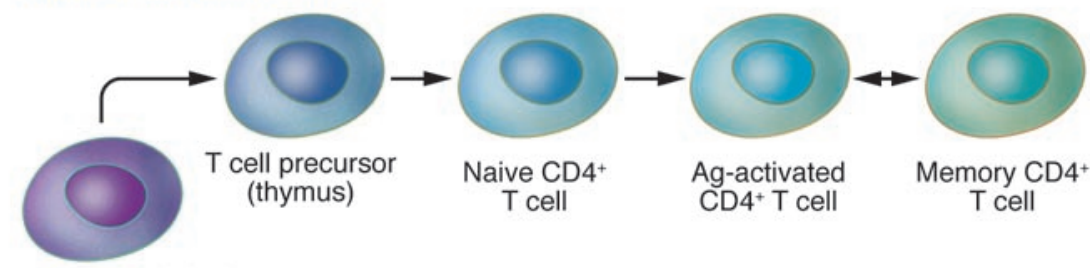

Bone marrow-derived

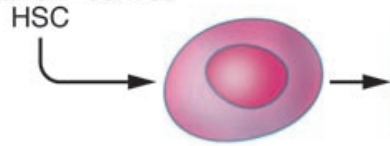

Precursor of rbc, wbc, and platelet

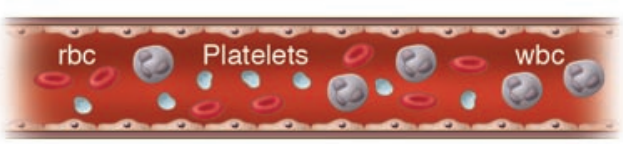

Peripheral blood

\section{B HIV infection}

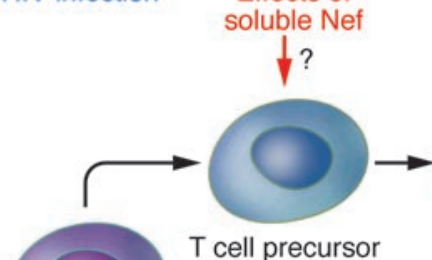

(thymus)

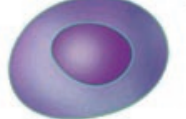

Bone marrow-derived
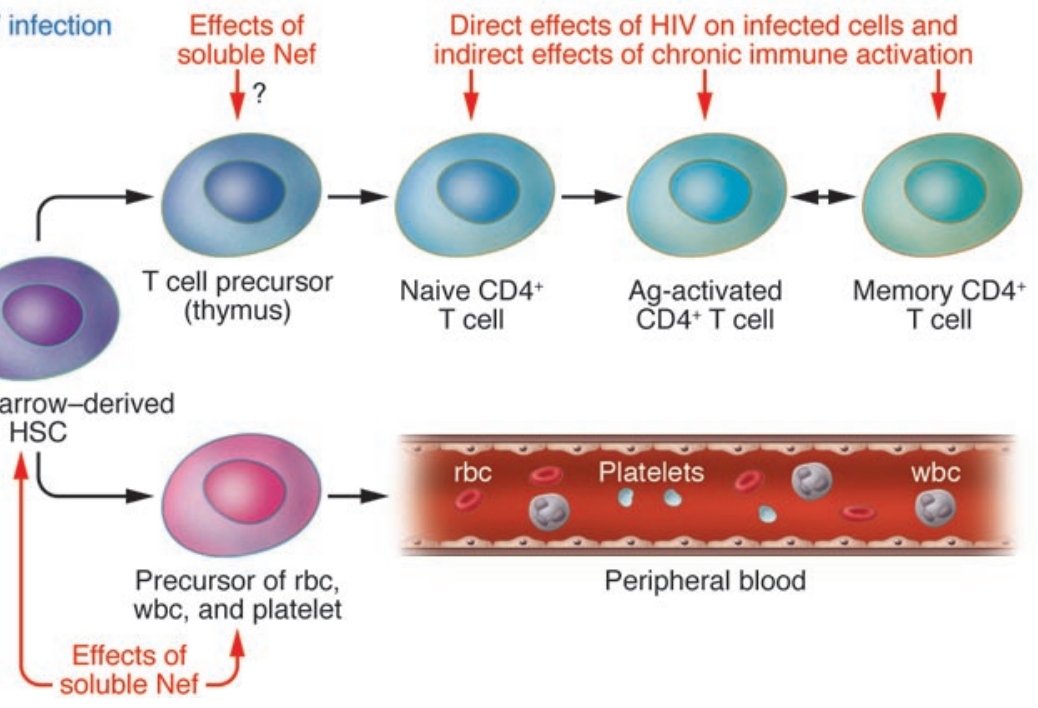

Peripheral blood several studies indicate that they are not susceptible to HIV-1 infection (7-9). Alternative mechanisms that were proposed - although without definitive experimental evidence include an effect of HIV-1 proteins on uninfected HSCs as well as the hematosuppressive potential of certain proinflammatory cytokines that are produced at high levels during HIV infection (10-13).

\section{Impaired STAT5 and HSC function in SIV-infected macaques}

The study from Prost and colleagues in this issue of the JCI (14) describes a series of experiments aimed at elucidating the mechanisms causing the hematopoietic abnormalities associated with HIV infection and AIDS. The main conclusion is that the extracellular viral accessory protein Negative factor (Nef) causes hyperactivation of $\operatorname{PPAR} \gamma$, that in turn reduces the expression of the STAT5A/B transcription factors, with consequent functional defects of HSCs (Figure 2). In the first set of experiments, the authors studied SIVmac251-infected macaques and confirmed the existence of three key features of HIV-1-associated defective hematopoiesis: (a) the number of CD34 $4^{+}$HSCs remains normal; (b) the clonogenic potential of these cells declines progressively over the course of infection; and (c) these cells are not infected by the virus. Next, they showed that the HSC defect correlates with low levels of STAT5A and STAT5B expression and, importantly, that overexpression of STAT5B in CD34+ cells by a lentiviral vector rescues the clonogenic function. The role of STAT5A/B in regulating the proliferation, differentiation, and survival of HSCs is well established (15), and the current results reported by Prost and colleagues are consistent with data from murine models showing that knockdown of either STAT5A or STAT5B causes anemia and erythroid defects (16), whereas double knockout of STAT5A and STAT5B proves to be highly anemic in utero and perinatally lethal (17).

\section{Figure 1}

Pathogenesis of the hematological abnormalities associated with HIV infection. (A) Schematic demonstrating how bone marrow-derived HSCs develop into precursors of the three main blood cell lineages (erythroid cells, myeloid cells, and platelets) as well as into the T cell precursors that will mature in the thymus and create the pool of circulating naive and memory CD4+ $\mathrm{T}$ cells. Ag, antigen. (B) Schematic demonstrating how the soluble viral accessory protein Nef may affect the ability of HSCs to maintain normal levels of peripheral blood cells, based on the new findings of Prost and coworkers reported in this issue of the $\mathrm{JCl}$ (14). In addition, Nef may decrease the production of $\mathrm{T}$ cell precursors, thus contributing to the peripheral $\mathrm{CD} 4^{+} \mathrm{T}$ cell depletion observed in HIV-infected individuals that is caused by both the direct effects of HIV on infected cells and the indirect effects of chronic immune activation.

\section{A role for Nef in impaired hematopoiesis}

Surprisingly, the Prost study (14) shows that extracellular, soluble forms of the HIV and SIVmac Nef proteins may downmodulate STAT5 expression by activating the transcriptional suppressor PPAR $\gamma$, thus providing a new mechanism to explain the HIV-associated HSC defects (Figure 2B). Of note, this inhibitory effect of soluble Nef on the clonogenic potential of progenitor cells (which the authors elegantly map to a central region of HIV-1 Nef between amino acids 66 and 97) might explain why the hematological abnormalities of HIV-infected patients are correlated with disease progression even though HSCs are not directly infected by the virus. Nef is an early accessory protein of immunodeficiency viruses that is required for efficient viral persistence and strongly accelerates disease progression in HIV-1-infected humans and in experimentally SIV-infected rhesus macaques (18). Nef performs a striking variety of activities that allow the virus to spread effi- 
A

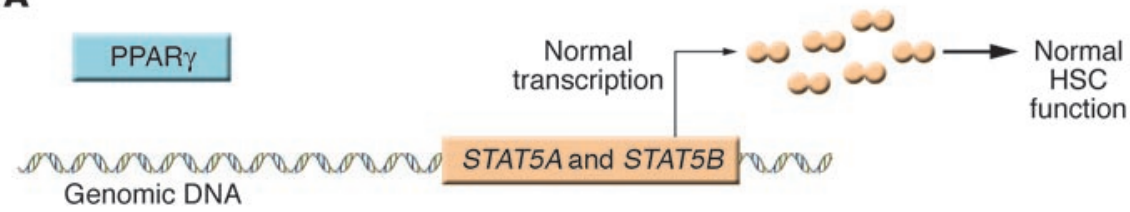

B

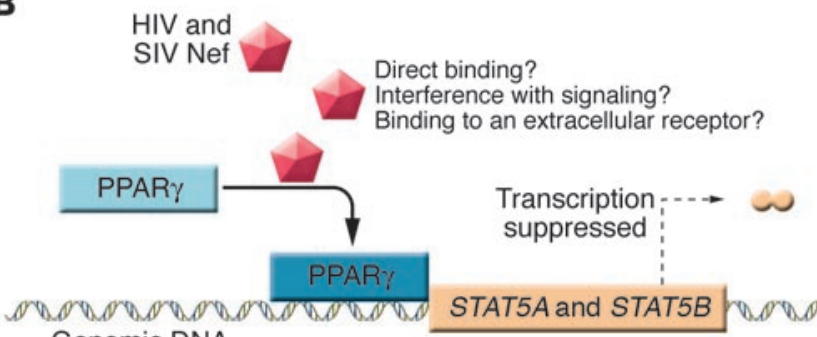

Genomic DNA

\section{Figure 2}

Nef-mediated suppression of the clonogenic potential of HSCs. (A) HSC clonogenic potential is dependent on transcriptional activation of STAT5A/B. (B) As reported in the current study by Prost et al. (14), in the setting of HIV and SIVmac infection, Nef induces upregulation of PPAR $\gamma$, which in turn suppresses the transcription of STAT5A/B, thus causing a reduction of the function and clonogenic potential of HSCs. ciently in the infected host and to evade the immune system, including downmodulation of CD4, MHC class I, and in many cases CD3 (most SIV strains and HIV-2, but not HIV-1 and its simian precursors), upregulation of the invariant chain associated with immature MHC class II complexes, and enhancement of viral infectivity and replication (18, 19). Furthermore, Nef interacts with various kinases, modulates cell signaling pathways, and might alter cytokine secretion (20). All these activities of Nef require an intact $\mathrm{N}$-terminal myristoylation signal and are exerted within the virally infected cell. However, Nef is also found in the extracellular environment and reaches concentrations of up to $10 \mathrm{ng} / \mathrm{ml}$ in sera of HIV-1-infected individuals (21). Reported effects of extracellular Nef include activation of STAT1 and STAT3 in macrophages (22), induction of apoptosis (23), and immunoglobulin class switching in bystander B cells (24). However, the true in vivo significance of these findings is difficult to assess as the above mentioned results were often obtained using rather artificial experimental conditions characterized by exceptionally high levels of Nef usually produced by bacteria. In this regard, Prost and coworkers (14) demonstrate that sera from SIVmac251-infected macaques can directly suppress STAT5A/B expression and cause HSC dysfunction (and that these effects are reduced by anti-Nef antibodies). These latter results not only support the possibility that the Nef-dependent STAT5A/B suppression occurs at "physiologic" concentrations of this protein, but also raise the question of whether humoral immune responses to Nef, which are detectable in the majority of HIV-1infected individuals (25), may prevent the development of hematopoietic dysfunction.

\section{Nef-mediated suppression of hematopoiesis: viral immune evasion strategy or suboptimal virus-host adaptation?}

As mentioned above, the current study (14) might explain why the severity of the hematologic abnormalities correlates with the levels of virus replication and why functional hematopoiesis is restored by HAART. The observation that extracellular Nef impairs the function of $\mathrm{CD} 34^{+}$progenitor cells in trans also clarifies why HIV-1 does not need to directly infect HSCs to cause hematological abnormalities. However, new interesting questions are raised by the observed results. For example, Prost et al. show that peptides corresponding to residues 66-97 of the central core region of HIV-1 $\mathrm{Nef}$ are sufficient to reproduce the defect of HSC function. Although this region is relatively well conserved, the HIV-1 and SIVmac251 Nef sequences differ in 9 of the 31 amino acid residues. Those of other SIVs are frequently even more divergent and - in contrast to HIV-1 Nef proteins - often do not contain an intact Src homology 3-binding (SH3-binding) domain in this region, raising the question of whether this Nef activity is conserved among the different groups of primate lentiviruses. This question is crucial as SIV infection of natural hosts such as the Sooty mangabeys or African green monkeys is not associated with immunodeficiency or impaired bone marrow function despite chronically high levels of virus replication (26). From this perspective, it will be interesting to elucidate whether this effect of Nef is mainly an indicator of suboptimal virus-host adap- tation in the evolutionarily recent human and experimental rhesus macaque hosts or indeed a conserved strategy of primate lentiviruses to evade the host immune system by suppressing the clonogenic potential of HSCs.

\section{Perspectives}

While the current study by Prost and colleagues (14) is of great interest, much work will be required to define the mechanism by which extracellular Nef induces PPAR $\gamma$ and to determine whether the levels of Nef in blood and bone marrow are sufficient to impair HSC function during HIV infection. It will also be important to better understand (a) to what extent this impaired hematopoiesis contributes to the $\mathrm{T}$ cell immunodeficiency associated with progression to AIDS, and (b) how these hematological defects relate to the state of chronic hyperimmune activation that seems to drive the loss of $\mathrm{CD} 4^{+} \mathrm{T}$ cells and progression to immunodeficiency in HIVinfected individuals. As STAT5 signaling plays a critical role in $\mathrm{T}$ cell development and function (27), an additional question is whether Nef also affects $\mathrm{T}$ cell homeostasis by suppressing STAT5A/B function in other, more mature $T$ cell subsets (28). From a clinical point of view, the results of Prost and colleagues may potentially identify Nef and/or PPAR $\gamma$ as target for new therapeutic approaches aimed at preventing or correcting the hematologic abnormalities associated with HIV-1 infection. On the other hand, the identification of PPAR $\gamma$ as a suppressor of HSC function might have implications for the use of PPAR $\gamma$ agonists, such as glitazones, in the treatment of diabetes. 


\section{Acknowledgments}

F. Kirchhoff thanks the Wilhelm-Sander Foundation, the Deutsche Forschungsgemeinschaft, and the NIH (R01AI067057) for support.

Address correspondence to: Frank Kirchhoff, Institute of Virology, University of Ulm, Albert-Einstein-Allee 11, 89081 Ulm, Germany. Phone: 49-731-50065109; Fax: 49-731-50065131; E-mail: frank.kirchhoff@uniklinik-ulm.de. Or to: Guido Silvestri, University of Pennsylvania School of Medicine, 705 Stellar-Chance Laboratories, 422 Curie Boulevard, Philadelphia, Pennsylvania 19143, USA. Phone: (215) 5735363; Fax: (215) 573-5366; E-mail: gsilvest@ mail.med.upenn.edu.

1. Sodora, D.L., and Silvestri, G. 2008. Immune activation and AIDS pathogenesis. AIDS. 22:439-446.

2. Moses, A., Nelson, J., and Bagby, G.C., Jr. 1998. The influence of human immunodeficiency virus-1 on hematopoiesis. Blood. 91:1479-1495.

3. Marandin, A., et al. 1996. Loss of primitive hematopoietic progenitors in patients with human immunodeficiency virus infection. Blood. 88:4568-4578.

4. Sloand, E.M., et al. 1997. Secondary colony formation after long-term bone marrow culture using peripheral blood and bone marrow of HIV-infected patients. AIDS. 11:1547-1553

5. Isgrò, A., et al. 2000. Recovery of hematopoietic activity in bone marrow from human immunodeficiency virus type 1 -infected patients during highly active antiretroviral therapy. AIDS Res. Hum. Retroviruses. 16:1471-1479.
6. Baillou, C., et al. 2003. Highly active antiretroviral therapy corrects hematopoiesis in HIV-1 infected patients: interest for peripheral blood stem cellbased gene therapy. AIDS. 17:563-574.

7. Koka, P.S., Jamieson, B.D., Brooks, D.G., and Zack, J.A. 1999. Human immunodeficiency virus type 1induced hematopoietic inhibition is independent of productive infection of progenitor cells in vivo. J. Virol. 73:9089-9097.

8. Weichold, F.F., et al. 1998. Neither human immunodeficiency virus-1 (HIV-1) nor HIV-2 infects most-primitive human hematopoietic stem cells as assessed in long-term bone marrow cultures. Blood. 91:907-915.

9. Zhang, J., Scadden, D.T., and Crumpacker, C.S 2007. Primitive hematopoietic cells resist HIV-1 infection via p21. J. Clin. Invest. 117:473-481.

10. Calenda, V., Graber, P., Delamarter,J.F., and Chermann, J.C. 1994. Involvement of HIV nef protein in abnormal hematopoiesis in AIDS: in vitro study on bone marrow progenitor cells. Eur. J. Haematol. 52:103-107.

11. Reinhold, D., Wrenger, S., Kahne, T., and Ansorge, S. 1999. HIV-1 Tat: immunosuppression via TGFbeta1 induction. Immunol. Today . 20:384-385.

12. Sugiura, K., Oyaizu, N., Pahwa, R., Kalyanaraman, V.S., and Pahwa, S. 1992. Effect of human immunodeficiency virus-1 envelope glycoprotein on in vitro hematopoiesis of umbilical cord blood. Blood. 80:1463-1469.

13. Maciejewski, J.P., Weichold, F.F., and Young, N.S. 1994. HIV-1 suppression of hematopoiesis in vitro mediated by envelope glycoprotein and TNF-alpha. J. Immunol. 153:4303-4310.

14. Prost, S., et al. 2008. Human and simian immunodeficiency viruses deregulate early hematopoiesis through a Nef/PPAR $\gamma /$ STAT5 signaling pathway in macaques. J. Clin. Invest. 118:1765-1775.

15. Bunting, K.D. 2007. STAT5 signaling in nor$\mathrm{mal}$ and pathologic hematopoiesis. Front. Biosci. 12:2807-2820.

16. Socolovsky, M., Fallon, A.E., Wang, S., Brugnara, C., and Lodish, H.F. 1999. Fetal anemia and apoptosis of

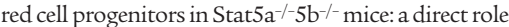

for Stat5 in Bcl-X(L) induction. Cell. 98:181-191.

17. Cui, Y., et al. 2004. Inactivation of Stat5 in mouse mammary epithelium during pregnancy reveals distinct functions in cell proliferation, survival, and differentiation. Mol. Cell. Biol. 24:8037-8047.

18. Foster, J.L., and Garcia, J.V. 2007. Role of Nef in HIV-1 replication and pathogenesis. Adv. Pharmacol. 55:389-409.

19. Schindler, M., et al. 2006. Nef-mediated suppression of $\mathrm{T}$ cell activation was lost in a lentiviral lineage that gave rise to HIV-1. Cell. 125:1055-1067.

20. Renkema, G.H., and Saksela, K. 2000. Interactions of HIV-1 NEF with cellular signal transducing proteins. Front. Biosci. 5:D268-D283.

21. Fujii, Y., Otake, K., Tashiro, M., and Adachi, A. 1996. Soluble Nef antigen of HIV-1 is cytotoxic for human CD4+ T cells. FEBS Lett. 393:93-96.

22. Federico, M., et al. 2001. HIV-1 Nef activates STAT1 in human monocytes/macrophages through the release of soluble factors. Blood. 98:2752-2761.

23. Okada, H., Takei, R., and Tashiro, M. 1997. HIV-1 Nef protein-induced apoptotic cytolysis of a broad spectrum of uninfected human blood cells independently of CD95(Fas). FEBS Lett. 414:603-606.

24. Qiao, X., et al. 2006. Human immunodeficiency virus 1 Nef suppresses CD40-dependent immunoglobulin class switching in bystander B cells. Nat. Immunol. 7:302-310.

25. Cheingsong-Popov, R. 1990. Antibodies to HIV-1 nef (p27): prevalence, significant and relationship to seroconversion. AIDS Res. Hum. Retroviruses. 6:1099-1105.

26. Silvestri, G., Paiardini, M., Pandrea, I., Lederman, M.M., and Sodora, D.L. 2007. Understanding the benign nature of SIV infection in natural hosts. J. Clin. Invest. 117:3148-3154.

27. Burchill, M.A, Yang, J., Vang, K.B., and Farrar, M.A. 2007. Interleukin-2 receptor signaling in regulatory $\mathrm{T}$ cell development and homeostasis. Immunol. Lett. 114:1-8.

28. Ye, P., Kirschner, D.E., and Kourtis, A.P. 2004. The thymus during HIV disease: role in pathogenesis and in immune recovery. Curr. HIV Res. 2:177-183.

\title{
Immunosuppression in islet transplantation
}

\section{Tom Van Belle and Matthias von Herrath}

La Jolla Institute for Allergy and Immunology, La Jolla, California, USA.

\begin{abstract}
Islet transplantation can temporarily cure type 1 diabetes mellitus (T1DM) but requires simultaneous immunosuppression to avoid allograft rejection. In this issue of the JCI, Monti et al. report that immune conditioning via use of the Edmonton protocol - a treatment approach in which T1DM patients infused with pancreatic islets from multiple cadaveric donors simultaneously receive immunosuppressive drugs - results in lymphopenia that is associated with elevated serum levels of the homeostatic cytokines IL-7 and IL-15, which causes in vivo expansion of the autoreactive $\mathrm{CD8}^{+} \mathrm{T}$ cell population (see the related article beginning on page 1806). Reemergence of autoreactivity is likely the main culprit underlying long-term islet graft failure, and new strategies will need to be tested to circumvent this homeostatic expansion and recurrent autoreactivity.
\end{abstract}

Nonstandard abbreviations used: GAD, glutamic acid decarboxylase; MMF, mycophenolate mofetil; T1DM, type 1 diabetes mellitus.

Conflict of interest: The authors have declared that no conflict of interest exists.

Citation for this article: J. Clin. Invest. 118:1625-1628 (2008). doi:10.1172/JCI35639.

\section{Autoimmunity, lymphopenia, and homeostasis}

Autoimmunity develops when immune cells attack and destroy our own cells. For instance, type 1 diabetes mellitus (T1DM) is a disease caused by immune-mediated destruction of the insulin-secreting pancreatic $\beta$ cells, leading to hyperglycemia. The immediate consequences of untreated T1DM (e.g., diabetic ketoacidosis) can be fatal, and, despite insulin therapy, long-term vascular complications can significantly affect life expectancy. Autoreactive T cells specific for $\beta$ cell autoantigens are present in most individuals, but are usually kept under control by peripheral tolerance mechanisms or have a regulatory phenotype (1). It is now well established that T1DM is the result of interactions between susceptibility genes (especially those within the HLA region) and probably several environmental factors (2). The highest-risk genotypes, HLA-DR3 or HLA-DR4 class II alleles, can be responsible for the development of anti-islet autoimmunity via production of autoantibodies to insulin; to glutamic acid 